\title{
DIMENSIONES DEL TRABAJO DOCENTE: UNA PROPUESTA DE ABORDAGE DEL MALESTAR Y EL SUFRIMIENTO PSIQUICO DE LOS DOCENTES EN LA ARGENTINA
}

\author{
Deolidia MartineZ* \\ Marité Collazo** \\ MANUEL Liss $^{* * *}$
}

\begin{abstract}
RESUMEN: La presente comunicación esta basada en estudios y trabajos de campo realizados a partir del convenio de cooperación entre la APBA y CTERA. Lleva 10 años de trabajo asentado en el programa de investigación "Salud en la escuela" del Instituto de Investigaciones Marina Vilte. El programa de investigación, con sus múltiples actividades, nos ha permitido trabajar con regularidad en todas las regiones del país con grupos de docentes de todos los niveles del sistema educativo. Nos hemos concentrado, particularmente, en el estudio teórico del análisis del proceso de trabajo docente y en el seguimiento de las singulares manifestaciones del sufrimiento psíquico en los docentes.
\end{abstract}

Palabras clave: Sufrimiento psíquico. Malestar docente. Factores de riesgo psíquico. Trabajo docente. Escuela pública.

DIMENSIONS OF TEACHERS' WORK: A PROPOSAL TO APPROACH TEACHERS' DISCOMFORT AND PSYCHIC SUFFERING IN ARGENTINA

ABSTRACT: This report is based on studies and fieldwork carried out following the cooperation agreement between APBA and CTERA. It

\footnotetext{
* Licenciada en Psicología y investigadora del Instituto de Investigaciones Marina Vilte (CTERA, Argentina). E-mail: deolidia@fibertel.com.ar

** Licenciada en Psicología, miembro de la Asociación de Psicólogos de Buenos Aires y investigadora del Instituto de Investigaciones Marina Vilte. E-mail: maritecollazo@gmail.com

*** Licenciado en Psicología, miembro de la Asociación de Psicólogos de Buenos Aires y investigador del Instituto de Investigaciones Marina Vilte.E-mail: mliss@fibertel.com.ar
} 
Dimensiones del trabajo docente: una propuesta de abordage del malestar...

presents ten years of work consolidated in the research program "Salud en la escuela" (Health in schools) of the Marina Vilte Research Institute. This research program, with its multiple activities, has allowed us to work regularly in all the regions of the country with groups of teachers from all the levels of the education system. We particularly focused on the theoretical analysis of the process of teaching and on monitoring unique manifestations of psychological suffering in teachers.

Key words: Psychological suffering. Teachers' discomfort. Psychological risk factors. Teachers' work. Public schools.

\section{Introducción}

$\mathcal{L}$

a enseñanza tiene una imagen social de actividad laboral sin riesgos, que fue cambiando en los últimos años, mal remunerada pero con algunas ventajas en comparación a otros trabajos, produciendo invisibilidad de factores que generan malestar y sufrimiento.

La realidad y la experiencia histórica procesada en diversas investigaciones señalan la existencia de fatiga residual, malestar docente con sufrimiento psíquico (depresiones, angustia, ataques de pánico, procesos psicóticos) y diversas formas de padecimientos psicofísicos que se manifiestan en la pérdida de la voz, dolores y contracturas en espalda, cuello y extremidades, úlceras gástricas e hipertensión, las más comunes. Últimamente se registra diabetes por stress con una frecuencia de alerta.

Al ser dolencias que se generan a lo largo de cierto tiempo de trabajo, son menos evidentes que un accidente laboral y muchas veces se perciben - subjetiva y socialmente - como problemas personales.

Un equívoco muy común se expresa en la "culpabilización de la víctima”, instalado como mecanismo socio-burocrático emisor de inadecuados juicios técnico-administrativos, descalificadores de los docentes, sobre problemas de salud, por personas o autoridades no competentes en el tema.

Un tema significativo son los bajos salarios porque tienen un efecto en cadena; la preocupación pasaría por la subsistencia y no por la existencia. Así el docente piensa prioritariamente como llegar a fin de mes para conseguir los alimentos que el cuerpo requiere para funcionar 
y no en otro tipo de alimento que apunta a la calidad expresiva y complejización del aparato psíquico en la vida social y cultural.

La modernidad construyó esta existencia escolar. Luego ya no hay - nadie propone - cómo sostenerla, "se" deja caer la escuela en su materialidad... el imaginario social la sostiene... un sujeto colectivo la va transformando sin hablar, sin explicar; nosotros, con distancia de observador, interpretamos y nos involucramos en ese mismo sujeto histórico que constituimos.

Esta investigación avanza con la definición de dimensiones observables de los factores de riesgo, instrumento indispensable para la aplicación en terreno por los propios sujetos involucrados. El contenido conceptual de las dimensiones se agrupada en tres categorías: a) dimensiones subjetivas y afectivas, b) dimensiones histórico-institucionales y c) dimensiones del proceso de trabajo docente. En este artículo exponemos una síntesis del contenido de las dimensiones a y c.

El objetivo de este ensayo es presentar una estructura conceptual y metodológica que sustente las prácticas del programa de investigación "Salud en la escuela" del Instituto de Investigaciones Pedagógicas Marina Vilte, estudios y trabajos de campo realizados a partir del convenio de cooperación entre APBA y CTERA. Lleva 10 años de trabajo asentado en el programa de investigación citado.

\section{Antecedentes: marco referencial}

El programa de investigación, con sus múltiples actividades, nos ha permitido trabajar con regularidad en todas las regiones del país con grupos de docentes de todos los niveles y modalidades del sistema educativo.

Un hito importante fue la realización de las Jornadas de Malestar Docente, desde 1999 a la fecha coordinamos seis eventos, en los que docentes y profesionales discutieron sus estudios, ensayos y reflexiones sobre este "síndrome" en el cual - a partir de su definición por José Manuel Esteve (1987) - nos hemos concentrado; particularmente en el estudio teórico del análisis del proceso de trabajo docente y en el seguimiento de las singulares manifestaciones del sufrimiento psíquico en los docentes.

Intercambios entre grupos de docentes, profesionales de la salud laboral e investigadores del trabajo docente en el seno de la Red Estrado, nos aportaron reflexiones que fueron poniendo palabras a lo que 
Dimensiones del trabajo docente: una propuesta de abordage del malestar...

escuchábamos tras lo oído, ese rumor "que era manera de decir". En un primer momento fue designado como malestar docente, termino que fue apropiado por los docentes y junto a ellos le fuimos dando categoría y especificidad propia. La unidad teoría-práctica avanzó penetrando nuestra tarea. Esa especificidad nos fue llevando a percibir que tras ese rumor había padecimiento, sufrimiento psíquico que se expresaba a través de múltiples manifestaciones. Sobre ese sufrimiento es de lo que hablamos.

El campo educativo se encuentra atravesado por un complejo entramado de significaciones sociales. Estas producciones de sentido logran cristalizarse a partir de su consolidación y reproducción a través de mitos, emblemas, rituales que sostienen a una sociedad. Esto es lo que Castoriadis (1983) denomina imaginario social instituido. Mitos, emblemas, ritos que, al no ser cuestionados, provocan síntomas en quienes lo sostienen.

Una sociedad es una construcción, se mantiene por la consolidación y reproducción de sus producciones de sentido. Esta inventa sus significaciones, las cuales organizan, orientan y dirigen la vida de los individuos que la constituyen.

La escuela como institución de la modernidad se consolida en el imaginario colectivo, naturaliza su presencia en el contexto social y no sólo justifica su existencia sino que su universalidad la hace necesaria como la vida misma. Los docentes que en ella trabajan están asimilados de tal forma que cuando se dice "la escuela es" o "a la escuela le falta” se personaliza en esa palabra a los que la realizan, materializándolos en ese imaginario que da por supuesto que "la escuela" contiene "los docentes y su circunstancia" y de los que realmente no se habla. Esta situación genera un circuito cerrado que dificulta la introducción de otros discursos, aunque no necesariamente éstos puedan cuestionar lo instituido: ¿Un lugar de relativo poder? ¿Un rol, un status social? ¿Un trabajo asalariado? ¿Qué ha delegado la sociedad en los docentes cautivos en "la escuela"?

Como dice Castoriadis (1983), que si bien desde el imaginario social se estructuran acontecimientos, ideales, hay algo que se le escapa, es la dimensión "del ser por hacerse" motor inagotable de la autotransformación, en lucha constante con la alienación basada en la hiper-adaptación a lo instituido. 
La escuela es una construcción social, que mantiene el orden social vigente en cada momento histórico. Vemos así a la escuela como un instrumento para la consolidación y perpetuación de ciertas normas, valores, ideas, que permiten el funcionamiento de determinados modelos vinculares.

Pero al mismo tiempo en la escuela no se da sólo un movimiento de mera conservación de lo instituido, sino que se enfrenta con un movimiento transformador, instituyente, el cual inventa nuevos conjuntos de significaciones y da cuenta de las líneas de fuga que los deseos posibilitan con relación al disciplinamiento social. Este movimiento transformador es visto como amenazante por la sociedad.

\section{Dimensiones subjetivas y afectivas}

\section{Los vínculos en el trabajo docente}

Las relaciones entre sujetos están constituidas y sostenidas en los vínculos afectivos que desarrollan. La relación maestro-alumno (núcleo del proceso de trabajo y de la subjetividad docente) se constituye como vínculo en tanto establece un entramado afectivo reconocido - registrado - por ambos términos de la relación vincular.

El vínculo de trabajo se construye además con los pares, las actividades con los compañeros/as (con y sin funciones de conducción y responsabilidades institucionales) son al mismo tiempo tan determinantes como la relación con los alumnos, aunque de diferente signo y contenido.

Esta relación vincular docente-docente y su dimensión afectiva real están menos analizadas que la de docente-alumno; su registro individual y/o grupal es más difuso, a veces es sentido como una amenaza grupal e individual.

\section{El tema del acoso en los ámbitos laborales}

Diversas formas de abuso de autoridad de una persona sobre otra, en especial cuando hay una relación de poder o mandato de autoridad - explícito o implícito - entre ambos, la relación cotidiana se hace insoportable para el o la sometido/a. En nuestro país ya hay un fuero 
Dimensiones del trabajo docente: una propuesta de abordage del malestar...

civil-administrativo específico con una Fiscalía para hacer las denuncias. En otros países el conflicto de acoso ya está regulado con normas de control y sanciones específicas para el abusador (España, Inglaterra, Canadá).

En la situación de acoso: no hay relación entre pares con diversidad de funciones

Hay necesidad de dominación y anulación de la autonomía del otro para un beneficio personal. En las relaciones de trabajo, desde la construcción de la subjetividad, lo necesario es que se establezca una distancia óptima, de los docentes entre sí y con los alumnos, para el desarrollo de la actividad de enseñar y aprender entre sujetos.

Lo deseable es una relación afectiva apoyada en el reconocimiento de la diferencia y en la discriminación subjetiva entre yo y el otro

Muchos problemas en los grupos de trabajo, en las aulas y en la escuela, se generan en la dificultad de reconocer y sostener las diferencias y las igualdades. Tanto entre los docentes como de éstos con los alumnos.

Desde el punto de vista preventivo: es necesario crear un espacio y tiempo instituido entre pares "comprensivos" (con o sin autoridad institucional) para poder comunicarse y procesar, integradamente, lo didáctico-pedagógico y lo afectivo que sustenta el vínculo de trabajo.

\section{Dimensiones del proceso de trabajo docente}

Condiciones materiales de trabajo: edificios, instalaciones, medios e instrumentos de trabajo

\section{La naturalización del riesgo}

Hay algunos factores de riesgo físico como el ruido, interno y externo, la iluminación escasa, la falta de espacio de trabajo, hacinamiento en aulas y patios, la falta de espacios de reunión y sanitarios para docentes y otras carencias que provocan malestar, alteraciones 
emocionales y sensoriales en docentes y alumnos. Las condiciones materiales del local escolar con instalaciones eléctricas inseguras, ampliaciones de construcción precaria y la provisión de medios e instrumentos de trabajo, deficiente o inexistente, determinan resultados que empobrecen el trabajo docente y al sujeto que lo realiza. El riesgo es que éste puede o no tener conciencia de ello.

Tanto la invisibilización de los riesgos, como la insensibilización que se genera en los trabajadores como defensa son mecanismos psicosociales de las relaciones laborales que se han construido al interior de los procesos de trabajo en largo tiempo de negación de su existencia.

Esta situación se agrava cuando en el registro interno del sujeto docente hay una lectura del "deber-ser" garante de la integridad de los alumnos que "desmiente" sus propias situaciones de riesgo y desvalimiento.

\section{La relación con el conocimiento}

La actualización científica, pedagógica y de técnicas para la realización del trabajo es una necesidad muy sentida en esta época de cambios rápidos mundiales y locales; nuevas exigencias de la sociedad y la economía repercuten a nivel personal en la vida diaria.

El mundo del trabajo ha tenido una transformación acelerada por la globalización del mercado que llevó a cambios específicos por regiones y sectores laborales.

La subsistencia, "el ganarse la vida", tiene sorpresas frente a las que el sujeto puede no tener respuesta por falta de conocimiento.

La incertidumbre ante el mercado laboral afecta a docentes y alumnos, en tiempo presente y a futuro, a cada uno y a la relación vincular entre ambos.

El campo de la educación pública presenta diferencias y desventajas respecto de otros ámbitos laborales, con referencia a la capacitación y acceso permanente a centros de actualización para los trabajadores docentes en Argentina. Esta dejó de ser - en muchas jurisdicciones - una actividad gratuita en servicio, provista por los empleadores en forma útil, oportuna y accesible.

En la actualidad es una exigencia personal del docente, que lo lleva a realizar la capacitación obligatoria en horas no-laborales y/o una 
Dimensiones del trabajo docente: una propuesta de abordage del malestar...

actualización voluntaria - arancelada - en zonas y horarios no siempre accesibles. Las formas burocráticas-meritocráticas han dado un sentido mercantil a la mera acumulación de puntajes para ascensos y permanencia en el cargo.

Una deuda histórica con el sector laboral docente es actualizar el reconocimiento del valor del trabajo.

Los salarios no tienen una composición adecuada al trabajo real. Tanto el tiempo de realización (dentro o fuera del aula o de la escuela), la carga en número de alumnos, como las responsabilidades y la complejidad cada vez mayores de las tareas educativas no están remuneradas. No tienen una asignación de valor económico específico (sólo el cargo directivo).

El trabajo no-remunerado y no siempre "voluntario" es un factor de compromiso psicológico para el docente, ya analizado en otros títulos de las dimensiones observables (carga pública, prescripciones, organización del trabajo).

Desde el punto de mira político-social "el doble discurso es enloquecedor". Veamos la situación del trabajador de la educación: la contradicción entre el alto valor asignado a la educación y a los docentes en abstracto en los discursos oficiales y programas políticos - y las formas concretas de abandono de las escuelas, desfinanciamiento del sistema educativo y deterioro del valor adquisitivo del salario docente; como "doble discurso" es un factor de riesgo psíquico que está muchas veces sin registro por los mismos afectados.

De acuerdo a lo deficitario de sus ingresos respecto a la subsistencia personal-familiar y a la capacidad de lucha que esta situación provoca en cada docente y en su grupo de referencia sindical-orgánico, es el lugar de lo observable. La reacción con riesgo es la tendencia al aislamiento, la queja y la desidia. Las respuestas colectivas con búsqueda de cohesión y fuerza social dan contención individual y expresan un proyecto político a futuro para mejorar las condiciones de vida y de trabajo.

Respecto del trabajo en el sector público en Argentina, el proceso de trabajo docente tiene un núcleo afectivo central constitutivo de la relación pedagógica que potencia un sufrimiento con el otro. La pobreza de las familias y comunidad escolar, cuando se une a la propia, puede ser angustiante. En especial cuando la experiencia 
personal familiar del docente ha sido más solvente en anteriores etapas de la vida y el presente no anticipa un futuro con esperanza de cambio positivo.

Los seguros de riesgos de trabajo: el acceso a servicios de salud

El desfinanciamiento del Estado nacional y la privatización de los servicios públicos alcanzaron a la seguridad en el trabajo. Las empresas aseguradoras no satisfacen adecuadamente las necesidades de información, capacitación y prevención de los riesgos de trabajo en la escuela. Las prestaciones de accidentes y enfermedades profesionales se otorgan con algunos obstáculos, en especial respecto a su accesibilidad en el territorio de cada jurisdicción y a regulaciones internas de los servicios contratados que frecuentemente no facilitan la asistencia de los trabajadores. La falta de información completa y oportuna de los docentes, de sus derechos y de los procedimientos administrativos requeridos hace que, ante la necesidad y para no realizar trámites burocráticos, recurra a su obra social para asistencia de accidentes laborales y enfermedades profesionales, a las que no denuncia como tales. Hay un alarmante subregistro de accidentes de trabajo y enfermedades profesionales en el sector docente.

Efecto de situaciones de fatiga - ya analizadas - y a la falta de desarrollo de una cultura de la prevención del riesgo de trabajo en la escuela y del conocimiento de los mismos, los docentes tienen serias carencias en el cuidado a su salud laboral.

En este caso son factores de riesgo psíquico, la falta de información técnica y las decisiones impulsivas que, ante una urgencia, toma el trabajador - por sí mismo - sin atender a situaciones de mayor peligro a las que se expone él y los alumnos y compañeros de trabajo.

\section{La atención de salud y en especial de salud mental}

El acceso a servicios de salud públicos y de la obra social correspondiente, para control periódico y asistencia de enfermedades, es el único recurso con que cuenta el trabajador. Los servicios privados, en algunas oportunidades, son utilizados como posibilidad ante la carencia de oferta pública. 
Dimensiones del trabajo docente: una propuesta de abordage del malestar...

En el caso de la atención de salud mental, hay escasas posibilidades de atención gratuita y el acceso a la atención privada resulta un esfuerzo económico que sólo algunos pueden realizar. Cuando se canaliza a través de la obra social, el área de salud mental (nunca valorada suficientemente) es el servicio donde suelen implementarse políticas de ajuste, limitando los tratamientos en el tiempo y a veces en la modalidad; lo cual es fuente de angustia y ansiedad para muchos/as docentes que acceden a ellos luego de largas listas de espera. La opción es un corto tratamiento o medicación con controles periódicos. La licencia suele convertirse en el único "tratamiento".

El hábito de concurrir a servicios de salud en forma periódica es un factor de prevención que se valora en la enseñanza como contenido, aunque no se practica con la frecuencia necesaria. Tal es, por ejemplo, el control ginecológico anual o semestral muy poco realizado y el alto índice de cáncer cérvico-uterino y de mamas en las mujeres docentes. La prevención en temas buco-dentales es casi inexistente, provoca pérdida de piezas dentarias y mayores gastos cuando el tratamiento es de urgencia.

Una actitud activa de prevención en temas de salud es indicador de calidad de vida en todos los sectores laborales y sociales. En el caso docente, se une a temas curriculares universales que seguramente conoce y trabaja.

\section{Tiempos y espacios en el trabajo docente: el factor humano}

La organización del trabajo es tema central de análisis - por los cambios que aceleradamente se produjeron en su interior - en el último siglo de estudios psicosociales, socio-económicos, ergonómicos y de medicina laboral.

El tiempo y los espacios de realización de los procesos de trabajo pasaron a primer plano tanto para valorar la producción como para el diseño de las condiciones y medio ambiente de trabajo. El factor humano individual y colectivo toma importancia decisiva en las transformaciones económicas de la globalización, ya que éstas determinaron cambios en la organización del trabajo productivo en todas las etapas del proceso.

El trabajo docente presenta un considerable atraso relativo en su estudio, tanto en la administración pública como en el sector 
educación. La organización del trabajo docente no tiene desarrollada una entidad propia de análisis.

En nuestro país, el trabajo docente está condicionado a las exigencias de una organización escolar cada vez más precaria - con cambios que se producen "de-hecho" -, determinada por políticas presupuestarias deficitarias frente al incremento de la matricula y los cambios de estructura del sistema impuestos.

La organización de la vida laboral escolar, tanto para docentes como para alumnos, se constituye en un factor de riesgo al no tener en cuenta "lo humano" de la actividad cotidiana - como relación y vínculo inter-subjetivo - eje determinante de la actividad educativa.

La organización escolar está diseñada sobre un modelo de trabajo que aísla a cada grupo en tiempos y espacios áulicos cerrados, difíciles de cambiar.

Las reformas en la estructura del sistema educativo de la última década cercenaron tiempos y espacios de construcción colectiva, de análisis de la práctica y de diseño de alternativas pedagógicas, didácticas e institucionales que trabajosamente se habían conseguido en muchos años de lucha sindical.

Lógicas de organización diversas y a veces contrapuestas - en el sentido que históricamente se desarrollaron en aula, escuela y sistema educativo - "estallan" en la subjetividad docente cuando falta la reflexión crítica y la posibilidad de analizar colectivamente su construcción en cada espacio de trabajo.

La informalidad, la ambigüedad, el "arréglese como pueda con lo que tiene", frente a nuevas necesidades, demandas y exigencias sociales, se constituyen en contenidos del trabajo docente cotidiano y en factor de riesgo psíquico cuando no hay capacidad crítica y seguridad en la respuesta individual de cada docente y el grupo no está organizado para defenderse y producir cambios. Es una grave carencia en el diseño de las políticas educativas de nuestro país que remite a una dificultad casi estructural para desarrollar cambios normativos de organización escolar.

En términos concretos, el tiempo y espacio real de trabajo con frecuencia son insuficientes para el trabajo colectivo y la vida social en la escuela. Puede observarse: pocos o nulos espacios y tiempos de descanso para el docente en la jornada laboral. Inadecuada previsión de 
Dimensiones del trabajo docente: una propuesta de abordage del malestar...

tiempo para la coordinación de tareas ínter e intra-grupos docentes. Esta tarea se realiza en tiempos extras y fuera del local de trabajo, muchas veces con el agravante de, además, ser prescripta.

En ésta como en otras dimensiones a observar, es necesario valorar el esfuerzo colectivo por construir consensos internos en cada escuela, a fin de organizar el trabajo cotidiano de forma más saludable para todos, y así limitar un factor de riesgo psíquico en el grupo docente.

La falta de separación entre trabajo y descanso durante la jornada laboral y la carga de tareas que obliga llevar "pendientes" al hogar dificultan otra necesaria separación de ámbitos personales: el laboral y el familiar. Siempre ocasiona fatiga y conflicto esta intromisión que lesiona tiempos y espacios específicos en la vida cotidiana del docente.

La inadecuación permanente entre prescripción y trabajo real es un factor de riesgo en cualquier trabajo. Muy visible en el trabajo docente. La presión psicológica en el control burocrático de resultados de aprendizaje, el desconocimiento y falta de retroalimentación de procesos de trabajo por sus protagonistas lleva consigo una carga de sufrimiento psíquico visible: en signos de una fatiga residual difícil de reparar.

La organización del trabajo en el sector docente puede ser un factor de riesgo tanto por la desconsideración y desconocimiento de la jurisdicción responsable de su definición, como por la rigidez y desgaste que presenta una estructura organizativa ya estereotipada y sin sentido.

\section{El conflicto social entró en la escuela}

Cuando hay una disociación (separación) entre el adentro (escuela) y el afuera (mundo externo), la sociedad en general y el docente en particular pueden actuar bajo el supuesto engañoso que la escuela es un área libre de conflicto social.

La escuela pública es una institución social muy permeable al conflicto tanto urbano como rural. Históricamente constituida, cumple mandatos familiares y culturales desde hace siglos. Muchas veces hay negación de la situación y la demanda se expresa desconociendo la historia nacional y local.

Sin embargo, las instituciones educativas reproducen el modelo de relación vigente en el contexto social. Sostener esa disociación es un factor 
de riesgo psíquico, ya que un hecho de violencia en la escuela desestructura el imaginario de docentes y familias. Las cosas no pasan sólo afuera. Se rompe el mito de que dentro de la escuela estas situaciones "se manejan" o es un espacio público preservado de daño y delito.

"A la escuela no la van a tocar"... es una situación imprevisible... insostenible.

Reconocer que es posible que se manifieste en cualquier escuela es una posición realista. Anticipa formas de prevención para elaborar algunas estrategias defensivas útiles para el grupo, que ayudan a comprender la realidad social con anticipación de sentido para la comunidad.

Como factor de riesgo en el trabajo docente, la violencia social incide en grupos vulnerables por su rigidez y aislamiento prejuicioso. La escuela pública ha funcionado como centro de control social mucho tiempo, actualmente esa función está en crisis y la necesidad de un cambio de estrategia se impone. La incertidumbre frente a situaciones pendientes de un nuevo rumbo en construcción no se resuelve individualmente. El factor de riesgo tiene que controlarse en forma colectiva y organizada. Enfrentar la destitución del mito es traumático. El templo del saber violado y destruido, no se acepta fácilmente. Al docente perturba tanto el no-saber, como la falta de control sobre un espacio que cree propio y seguro como "mi escuela".

Malestar y sufrimiento psiquico: Lo no dicho que se escucha tras lo dicho lizado:

Algunas frases de docentes tomadas del trabajo de campo rea-

"Nunca había oído hablar de estos temas de este modo. De esto, así, no se habla en la escuela".

"A muchos les pasa lo mismo que a mí".

"Con el afán de educar, descuidamos...".

"No me quiero enterar de que algo está mal" lo no dicho es "y eso me hace mal...".

"Me siento a la intemperie". 
Dimensiones del trabajo docente: una propuesta de abordage del malestar...

"No nos creen por ser compañeros, esperan que se los digan las autoridades".

"Nos vemos todas locas".

"No se si seguir o no en el trabajo...".

"Ah... de eso no se habla". "Cada uno se salva como puede, a mi no me carguen problemas ajenos...".

"Durante el recreo intercambio algunas palabras con los colegas, pero no me involucro mucho en el tema, el tiempo es muy corto, 7 minutos de recreo, un suspiro y al aula nuevamente. Trato de no gastar mucho mis energías para mantenerme calma y sin sobresaltos, ya estoy cansada y bebo agua para evitar el sueño".

A partir de nombrar el malestar docente, lo reconocemos como una problemática compartida, no individual, que sufren los profesionales de la educación en el ejercicio de su función. Con amplitud de expresiones físicas y psíquicas hasta posibilitar su elaboración, es decir la posibilidad psíquica de salir de la tendencia inconciente a la repetición.

Contamos a favor con la pertenencia a un colectivo social, es una posibilidad que puede facilitar la expresión y elaboración del sufrimiento en la subjetividad de cada docente.

Desde el imaginario social, con sus correspondientes representaciones, se espera del docente determinados desempeños y conductas con relación a los otros y a sí mismo, por ejemplo: que se identifique con el alumno, reconozca los sentimientos que se ponen en juego en el otro, lo entienda, comprenda y contenga, exigiéndole al mismo tiempo que se abstenga de expresar sus preocupaciones, dificultades y emociones, siendo admitida la expresión sólo de sentimientos amorosos, desconociéndose que en las relaciones afectivas se ponen en juego tanto sentimientos amorosos como hostiles.

Al mismo tiempo el contexto institucional genera un doble discurso "esquizofrenizante"; por un lado el verbal, con el cual la sociedad y el sistema educativo piden calidad de enseñanza, y por el otro, el sin palabra, el de los hechos, en cuanto a las condiciones para realizar la tarea, ejercicio de poder que muestra el desprecio por las personas que la llevan a cabo, si bien está sostenido en argumentos presupuestarios, no podemos evitar ligarlo con la desvalorización que implica. 
Engrandecimiento y desvalorización de los docentes, doble discurso que hace síntoma. Ubicar al docente como garante de la formación y la integridad de los alumnos, a costa de su propia integridad, muchas veces solo preocupándose por los alumnos, produce la desmentida de sus situaciones de sufrimiento y de desamparo.

$\mathrm{Al}$ incluir lo psíquico, lo diferenciamos de lo psicosocial por la incorporación en el análisis de los contenidos inconcientes de la personalidad, ubicando al sujeto en una red vincular familiar y generacional que determina su singularidad. Singularidad que va a determinar el modo de relación vincular con el contexto, como así también los registros ligados a las vivencias de placer y sufrimiento.

Desde la teoría psicoanalítica referimos la estructuración psíquica del sujeto a partir de las tres instancias - Ello-Superyo-Yo (Freud, 1968) - y los mecanismos defensivos estructurantes.

Dicha estructuración psíquica, sostenida en el entramado de los vínculos afectivos primarios, contiene las matrices afectivas que se mantendrán inscriptas en el inconciente, y estarán presentes en el establecimiento de las relaciones familiares, sociales y laborales.

El sufrimiento psíquico, desencadenado en y por el trabajo, afecta al sujeto en su totalidad más allá del hábitat concreto, incluyendo su vida social y familiar. Sin embargo, frente a las mismas condiciones laborales adversas - salarios y calificación -, las respuestas de cada trabajador docente son diversas - singulares - según su personalidad y modo de defensa característico.

Este sufrimiento padecido por el sujeto puede ser reconocido como tal aunque no reconozca las causas que lo determinan; también pude no ser registrado subjetivamente o puede hacerse evidente sólo por la mirada de un otro.

En toda relación laboral se ponen en juego los afectos, mas aun en aquellas actividades como la docente donde lo vincular es el núcleo del proceso de trabajo, tanto en la relación docentes-alumnos, como de los docentes entre sí.

El inevitable involucramiento afectivo provoca el desarrollo de procesos concientes e inconcientes, que merecen tenerse en cuenta, entre ellos los despliegues transferenciales que son inconcientes.

Desde el imaginario social están presentes demandas implícitas y explicitas, se espera de la escuela y del docente representaciones 
Dimensiones del trabajo docente: una propuesta de abordage del malestar...

ideales, que en la mayoría de las veces no tienen que ver ni con la escuela ni con los docentes y lo que realmente pueden hacer cotidianamente en el trabajo.

Estas representaciones también están en el propio imaginario docente, lo que le lleva con frecuencia a generar conflictos con relación a su práctica docente. Tengamos en cuenta el mítico concepto de la vocación, la "cuna docente" etc.

Es fundamental diferenciar entre sujeto y función docente. La responsabilidad de la tarea docente esta ligada a la función, en el imaginario social como en muchos docentes queda distorsionada esta función ligándose con la identidad. El sujeto esta relacionado con el ser, en cambio la función es una acción del sujeto, un aspecto que se pone en funcionamiento en determinado momento: en una tarea concreta, en un atributo de su ser en el mundo.

Instado por las estructuras de regulación estatales, se ha alentado esta confusión entre sujeto y función, al servicio del sometimiento del docente ya que cuestionar condiciones laborales (función) lo llevaría a cuestionar la identidad inherente a la condición de sujeto.

Podemos pensar la función docente definida y sostenida fuertemente como vocación por gran cantidad de docentes, como un mecanismo identificatorio con el imaginario social que los ubican en un lugar ideal que define su ser. Lo real del trabajo no se toma en cuenta, está desconocido por la autoridad administrativa-burocrática.

La realidad ha impuesto un cambio en la función docente. Si antes podríamos definirla prioritariamente como función paterna, ya que generaba un orden de pautas y jerarquías, trasmisor de saber y conocimiento, se ha desplazado mayoritariamente a una función que podríamos definir como materna, ya que esta abocada a brindar sostén, cuidado, amparo y ternura, en un marco de satisfacer las necesidades básicas de alimentación y a propiciar la inclusión frente a la exclusión.

Para poder continuar con la tarea, muchos docentes apelan al mecanismo de la sobre- adaptación, generándoles un estado de máxima vulnerabilidad, ya que ante una falla de esta defensa sobrevienen sentimientos masivos de angustia, inseguridad, ataques de pánico, lo que predomina es la vivencia de que no es posible para el yo responder a las exigencias y las demandas, el yo se declara impotente. 
Se pone en juego la identidad subjetiva, no solo la función docente, entendiendo la identidad como un conjunto de representaciones que un sujeto posee de si, que corresponde a un sentimiento de si mismo.

Con la caída de los apuntalamientos psíquicos constituyentes de la identidad, sobrevienen la vivencia de desamparo y su concomitante angustia de aniquilación, que en la infancia se da por la amenaza de la perdida de amor de los padres y en la adultez por la vivencia de perdida de amor del super-yo.

Una de las funciones del super-yo es la de medir al yo con el ideal.

Responsabilidad social sobre los docentes

La demanda social sobre la escuela pública actualmente se constituye en una alta exigencia de responsabilidad social sobre los docentes, ante la carencia de respuesta oportuna y efectiva de los organismos de gobierno de la educación que se des-responsabilizan. Junto a otros trabajadores del sector público, la carga pública de su trabajo tiene un valor simbólico diferente al conjunto, de otro peso social.

Partimos de los conceptos de lo intimo como aquel espacio intrapsíquico del sujeto que remite al sí mismo, regido por el derecho al secreto; a lo privado como el espacio de la familia y por extensión el de la vida grupal y asociativa; espacio donde se producen los intercambios entre lo íntimo y lo publico. Lo público como espacio de la sociedad donde las tramas y las reglas deben ser comunes, conocidas, reconocidas y aprendidas, regidas por la transparencia. Por lo general, siendo el sufrimiento muchas veces originado en el espacio publico con lo cual debería estar regido por la transparencia de lo común que atraviesa a muchas personas iguales - pasa a ser tramitado en lo íntimo, regido por el secreto, constituyéndose en un problema de incumbencia individual, generalmente oculto.

Esta frase esta referida tanto hacia los alumnos como hacia sí mismo. El docente imbuido en su tarea, atrapado en una maraña de mandatos implícitos y explícitos, pasa por alto condiciones de trabajo real muy adversas. Queda más ligado a los mandatos del imaginario social, a lo que se espera de él, que a su deseo. 
Dimensiones del trabajo docente: una propuesta de abordage del malestar...

También hace mención a las resistencias de los docentes a verse como protagonistas de su propia historia. Esta frase de afirmación por la negación marca el concepto de autoridad que subyace y quién tiene la verdad en el trabajo docente. Es un buen ejemplo para ver la depositación en el otro de las propias ansiedades.

Es una definición, tomando el lenguaje popular, de cómo se ven y se sienten las docentes, no solamente en el ámbito laboral. Aquí es necesario leer con enfoque de género, ya que lo femenino de la docencia es portador de "la locura".

Esta reflexión de un docente da cuenta, por un lado, de su frustración y, por otro, de un nivel de salud que hace generar un interrogante acerca de su continuidad en ese trabajo. Este interrogante lo rescata de la alienación laboral que, sostenida en la sobre-adaptación, hace que muchos docentes no se pregunten por las condiciones laborales que padecen y si están dispuestos a tolerarlas...

Entre desistir y afrontar, el docente juega su malestar en el cotidiano laboral

Todo sujeto vive un permanente proceso de adaptación. Este proceso supone un conjunto de funciones psíquicas tendientes a la adaptación a la realidad, incluyendo la modificación de ésta - función llevada a cabo por el yo - en un inter-juego permanente entre lo pulsional, el super-yo y la realidad exterior, de allí que se plantea que la función yoica esta sometida a un triple vasallaje.

Ante exigencias desmedidas de la realidad exterior, o bien a propios ideales que deben ser cumplidos, se apela a la sobre-adaptación, donde hay un acoplamiento exagerado a las exigencias sin resistencia ni reflexión. Este mecanismo es muy común en los docentes.

Como manifestaciones diagnosticas devenidas del mecanismo de sobre-adaptación, se encuentran, entre otras:

- Temor al ocio sin reglas.

- Se establecen conductas adictivas a una imagen que permite valoración social, a expensas de un alto costo corporal y psíquico. 
- Dificultades en el registro de las percepciones de los receptores cenestésicos y propioceptivos.

- Trastornos del dormir y del soñar.

- El descanso es cuando "no se da más".

- El limite esta dado en el umbral del agotamiento total.

- En lugar de expresar emociones, muchas veces se producen modificaciones corporales, produciéndose un cuadro denominado "alexitimia", en el cual se tiene dificultad para designar las emociones tanto para los afectos dolorosos, como para los que expresan satisfacción y placer "anhedonia".

- Imposibilidad para describir estados afectivos.

- Incapacidad para distinguir un afecto de otro.

Quienes padecen estos trastornos suelen ser docentes realistas, prácticos, siempre muy preocupados por cumplir con exigencias y obligaciones, rechazan vincular sus afecciones a conflictos con la afectividad. La ansiedad, inquietud y excitación están ligadas a las exigencias de la tarea, a dificultades en el manejo de la agresión, escaso o nulo registro de las emociones y/o sensaciones corporales. Rechazo ante cualquier propuesta que vincule sus síntomas a un conflicto o sufrimiento psíquico personal.

Cuanto más visible - menos "natural" - resulte su sufrimiento, en mejores condiciones está el docente para trabajar en condiciones más saludables, accediendo a su subjetividad.

Este trabajo que expusimos está diseñado para que los docentes lo puedan utilizar y apropiarse de él. En la medida que logren identificar la propia angustia, ponerla en palabras y compartirla, baja el sufrimiento personal. Poner la palabra dificulta la constitución de síntomas: accidentes, depresiones, serios conflictos entre pares, irritabilidad.

Recebido em junho de 2009 e aprovado em julho de 2009. 
Dimensiones del trabajo docente: una propuesta de abordage del malestar...

\section{Referencias}

BARAMENDI, A. Del autoritarismo que sabemos conseguir. In: CONGRESO LATINOAMERICANO DE PSICOTERAPIA ANALÍTICA DE GRUPO, 11., 1994, Buenos Aires. Anais... Buenos Aires, 1994. v. 1.

CASTORIADIS, C. La institución imaginaria en la sociedad. Barcelona: Tusquets, 1983.

ESTEVE, J.M. El malestar docente. Barcelona: Paidós, 1987.

FREUD, S. Compendio del psicoanálisis: la naturaleza de lo psíquico. In: Freud, S. Obras completas. Madrid: Biblioteca Nueva, 1968. t. 3. 